\title{
The DarkSide Program
}

B. Rossi ${ }^{15,6, a}$, P. Agnes ${ }^{2}$, T. Alexander ${ }^{3}$, A. Alton ${ }^{4}$, K. Arisaka ${ }^{5}$, H.O. Back ${ }^{6}$, B. Baldin ${ }^{7}$, K. Biery ${ }^{7}$, G. Bonfini ${ }^{8}$, M. Bossa ${ }^{9}$, A. Brigatti ${ }^{10}$, J. Brodsk $y^{6}$, F. Budano ${ }^{11}$, F. Calaprice ${ }^{6}$, N. Canci ${ }^{5}$, A. Candela ${ }^{8}$, M. Cariello ${ }^{12}$, P. Cavalcante ${ }^{8}$, S. Catalanotti $^{15}$, A. Chavarria $^{13}$, A. Chepurnov ${ }^{14}$, A. G. Cocco $^{15}$, G. Covone ${ }^{15}$, D. D’Angelo ${ }^{10}$, M. D’ Incecco ${ }^{8}$, M. De Deo ${ }^{8}$, A. Derbin ${ }^{16}$, A. Devoto ${ }^{17}$, F. Di Eusanio ${ }^{6}$, E. Edkins ${ }^{18}$, A. Empl ${ }^{1}$, A. Fan ${ }^{5}$, G. Fiorillo ${ }^{15}$, K. Fomenko ${ }^{19}$, D. Franco ${ }^{2}$, F. Gabriele ${ }^{8}$, C. Galbiati ${ }^{6}$, A. Goretti ${ }^{6}$, L. Grandi ${ }^{13}$, M.Y. Guan ${ }^{20}$, Y. Guardincerri ${ }^{7}$, B. Hackett ${ }^{18}$, K. Herner ${ }^{7}$, E.V. Hungerford ${ }^{1}$, Al. Ianni ${ }^{8}$, An. Ianni ${ }^{6}$, C. Kendziora ${ }^{7}$, G. $\mathrm{Koh}^{6}$, D. Korablev ${ }^{19}$, G. Korga ${ }^{1}$, A. Kurlej ${ }^{3}$, P.X. Li $^{20}$, P. Lombardi ${ }^{10}$, S. Luitz $^{21}$, I. Machulin ${ }^{22}$, A. Mandarano ${ }^{9}$, S. Mari ${ }^{11}$, J. Maricic ${ }^{18}$, L. Marini ${ }^{11}$, C. J. Martoff ${ }^{23}$, P.D. Meyers ${ }^{6}$, D. Montanari ${ }^{7}$, M. Montuschi ${ }^{8}$, M.E. Monzani ${ }^{21}$, P. Musico ${ }^{12}$, S. Odrowski $^{8}$, M. Orsini ${ }^{8}$, F. Ortica ${ }^{24}$, L. Pagani ${ }^{12}$, M. Pallavicini ${ }^{12}$, E. Pantic ${ }^{5}$, L. Papp ${ }^{25}$, S. Parmeggiano ${ }^{10}$,

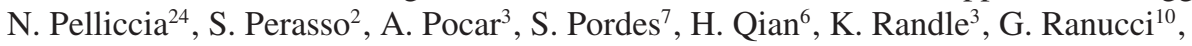

A. Razeto ${ }^{8}$, B. Reinhold ${ }^{18}$, A. Renshaw ${ }^{5}$, A. Romani ${ }^{24}$, N. Rossi ${ }^{8}$, S.D. Rountree ${ }^{25}$, D. Sablone ${ }^{1}$, R. Saldanha ${ }^{13}$, W. Sands ${ }^{6}$, E. Segreto ${ }^{8}$, E. Shields ${ }^{6}$, O. Smirnov ${ }^{19}$, A. Sotnikov ${ }^{19}$, C. Stanford ${ }^{6}$, Y. Suvorov ${ }^{5}$, R. Tartaglia ${ }^{8}$, J. Tatarowicz ${ }^{23}$, G. Testera $^{12}$,

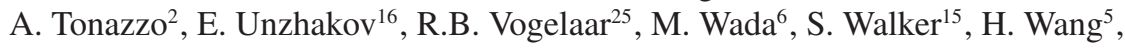
A. Watson ${ }^{23}$, S. Westerdale ${ }^{6}$, M. Wojcik ${ }^{26}$, X. Xiang ${ }^{6}$, J. Xu$^{6}$, C.G. Yang ${ }^{20}$, J. Yoo $^{7}$, S. Zavatarelli ${ }^{12}$, A. Zec ${ }^{3}$, C. Zhu ${ }^{6}$, and G. Zuzel ${ }^{26}$

${ }^{1}$ Department of Physics, University of Houston, Houston, TX 77204, USA

2 APC, Université Paris Diderot, Sorbonne Paris Cité, Paris 75205, France

${ }^{3}$ Physics Department, University of Massachusetts, Amherst, MA 01003, USA

${ }^{4}$ Physics and Astronomy Department, Augustana College, Sioux Falls, SD 57197, USA

${ }^{5}$ Physics and Astronomy Department, University of California, Los Angeles, CA 90095, USA

${ }^{6}$ Physics Department, Princeton University, Princeton, NJ 08544, USA

${ }^{7}$ Fermi National Accelerator Laboratory, Batavia, IL 60510, USA

${ }^{8}$ Laboratori Nazionali del Gran Sasso, Assergi (AQ) 67010, Italy

${ }^{9}$ Gran Sasso Science Institute, L'Aquila 67100, Italy

${ }^{10}$ Physics Department, Università degli Studi and INFN, Milano 20133, Italy

${ }^{11}$ Physics Department, Università degli Studi Roma Tre and INFN, Roma 00146, Italy

${ }^{12}$ Physics Department, Università degli Studi and INFN, Genova 16146, Italy

${ }^{13}$ Kavli Institute, Enrico Fermi Institute and Dept. of Physics, University of Chicago, Chicago, IL 60637, USA

${ }^{14}$ Skobeltsyn Institute of Nuclear Physics, Lomonosov Moscow State University, Moscow 119991, Russia

${ }^{15}$ Physics Department, Università degli Studi Federico II and INFN, Napoli 80126, Italy

${ }^{16}$ Saint Petersburg Nuclear Physics Institute, Gatchina 188350, Russia

${ }^{17}$ Physics Department, Università degli Studi and INFN, Cagliari 09042, Italy

${ }^{18}$ Department of Physics and Astronomy, University of Hawai'i, Honolulu, HI 96822, USA

${ }^{19}$ Joint Institute for Nuclear Research, Dubna 141980, Russia

${ }^{20}$ Institute of High Energy Physics, Beijing 100049, China

${ }^{21}$ SLAC National Accelerator Laboratory, Menlo Park, CA 94025, USA

\footnotetext{
${ }^{a}$ e-mail: rossib@princeton.edu
}

(C) The Authors, published by EDP Sciences. This is an Open Access article distributed under the terms of the Creative Commons Attribution License 4.0 (http://creativecommons.org/licenses/by/4.0/). 
${ }^{22}$ National Research Nuclear University Moscow Engineering Physics Institute, 115409 Moscow, Russia

${ }^{23}$ Physics Department, Temple University, Philadelphia, PA 19122, USA

${ }^{24}$ Chemistry, Biology and Biotechnology Department, Università degli Studi and INFN, Perugia 06123, Italy

${ }^{25}$ Physics Department, Virginia Tech, Blacksburg, VA 24061, USA

${ }^{26}$ Smoluchowski Institute of Physics, Jagiellonian University, Krakow 30059, Poland

Abstract. DarkSide-50 at Gran Sasso underground laboratory (LNGS), Italy, is a direct dark matter search experiment based on a liquid argon TPC. DS-50 has completed its first dark matter run using atmospheric argon as target. The detector performances and the results of the first physics run are presented in this proceeding.

\section{Introduction}

The majority of the matter and of the energy content of the universe seems to be dark. Cosmological and astronomical observations supported by the very recent results from the Plank satellite indicate that: the dark energy, responsible of the observed accelerated expansion of the cosmos, accounts for the $68 \%$ of the total budget; the dark matter, a yetundetected form of matter which forms the observed large-scale structures and galaxies, accounts for the $27 \%$, while the remaining $5 \%$ is composed by "ordinary" baryonic matter. Many theories beyond the standard model of particle physics predict possible candidates for dark matter particles and among those the Weakly Interacting Massive Particles (WIMPs). Although the strength of the interaction is supposed to be very small, the thermal motion of these WIMPs comprising the dark matter halo surrounding the galaxy and the earth is expected to result in WIMP-nuclear collisions of sufficient energy to be observable by very sensitive laboratory apparatus. The fact that dark matter exists is one of the strongest indications for physics beyond the standard model of particle physics. Its direct detection and characterization is a major experimental challenge of modern particle and astroparticle physics. The DarkSide project attempts to detect WIMP-induced nuclear recoils using twophase Argon time projection chambers (TPCs) with scalable, zero-background technology. The largest challenge in searching for dark matter is the suppression of the rate of background events to below the very low WIMP interaction rates (a few events per ton-year) to which current dark matter experiments are sensitive. The prompt scintillation light (S1) and the delayed proportional light signal from the charge (S2) are simultaneously measured in twophase TPCs and are used for a precise reconstruction of the event vertex and its multiplicity. Both signals are employed to suppress the backgrounds by target fiducialization and by rejection of multiple-scatter interactions (WIMPs are expected to only interact once). The charge-to-light ratio, S2/S1, is exploited to separate expected signal events, which are nuclear recoils (NR) from the dominant electronic recoil (ER) background and it allows discrimination up to a factor $10^{2}$. One reason that makes Argon a promising medium for dark matter searches is that it has a unique powerful feature, the pulse shape discrimination (PSD). Particles interacting in noble liquids induce excitation and ionization of the medium, leading to the emission of scintillation light whose time structure is strongly correlated with the nature of the interaction. This time dependence of the scintillation signal provides an additional way to detect rare nuclear recoil events, possibly induced by WIMPs. With sufficient photon statistics, PSD can allow discrimination of nuclear recoil events from electron-induced background events at better than $10^{8}[1,2]$.

The ultimate goal of DarkSide-50 is to conduct a background-free dark matter search with its 50-kg TPC filled with argon derived from underground sources (UAr) [3, 4], to reduce the 


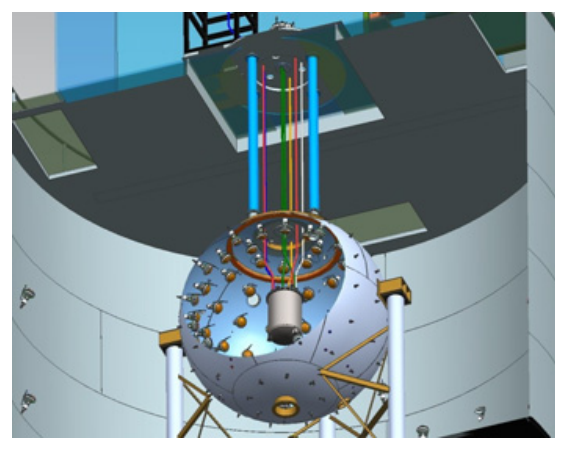

Figure 1. The detector system of DarkSide-50. The outermost dark gray cylinder is the WCD, the sphere is the LSV, and the gray cylinder at the centre of the sphere is the LAr TPC.

rate of ${ }^{39} \mathrm{Ar}$ decays in the active volume. The present exposure amounts to $(1422 \pm 67) \mathrm{kg}$ $\mathrm{d}$ using an initial fill of atmospheric argon, obtained while the final purification of the UAr supply was still in progress. Atmospheric argon contains approximately $1 \mathrm{~Bq} / \mathrm{kg}$ of cosmogenic 39Ar [5-7].

\section{The DarkSide-50 detector}

Figure 1 shows the experimental setup of the DarkSide-50 experiment composed by three detectors: the Liquid Argon Time Projection Chamber (LAr TPC) which is the dark matter detector, the organic Liquid Scintillator Veto (LSV), serving as shielding and as anticoincidence for radiogenic and cosmogenic neutrons, $\gamma$-rays and cosmic muons; and the Water Cherenkov Detector (WCD), serving as a shield and as anti-coincidence for cosmic muons. The detector system is located in Hall C of LNGS at a depth of 3800 meter-waterequivalent.

The WCD is an $11 \mathrm{~m}$-diameter, $10 \mathrm{~m}$-high cylindrical tank filled with high purity water. The inside surface of the tank is covered with a laminated Tyvek-polyethylene-Tyvek reflector. An array of 80 ETL 9351 8" PMTs, with $27 \%$ average quantum efficiency at $420 \mathrm{~nm}$, is mounted on the side and bottom of the water tank to detect Cherenkov photons produced by muons or other relativistic particles traversing the water.

The LSV is a 4 m-diameter stainless steel sphere filled with 30 tons of borated liquid scintillator. The scintillator consists of equal amounts of pseudocumene (PC) and trimethyl borate (TMB), with the wavelength shifter Diphenyloxazole (PPO) at a concentration of $3 \mathrm{~g} / \mathrm{L}$. The sphere is lined with Lumirror reflecting foils. An array of 110 Hamamatsu R5912 8 " PMTs, with low-radioactivity glass bulbs and high-quantum-efficiency photocathodes (37\% average QE at $408 \mathrm{~nm}$ ) is mounted on the inside surface of the sphere to detect scintillation photons. The neutron-capture reaction ${ }^{10} \mathrm{~B}(\mathrm{n}, \alpha){ }^{7} \mathrm{Li}$ makes the borated scintillator a very effective veto of neutron background [8]. A detailed description of the LAr TPC can be found in [9]. Additional information on the detectors employed in the DarkSide project can be found in [9-13].

\section{Data acquisition and event reconstruction}

The data acquisition system of DS-50 consists of two main sub-systems, handling the TPC and the vetoes respectively. The ground-referenced anodic signal from each of the thirtyeight TPC photomultiplier tube is first amplified by a cryogenic head-amplifier located in 


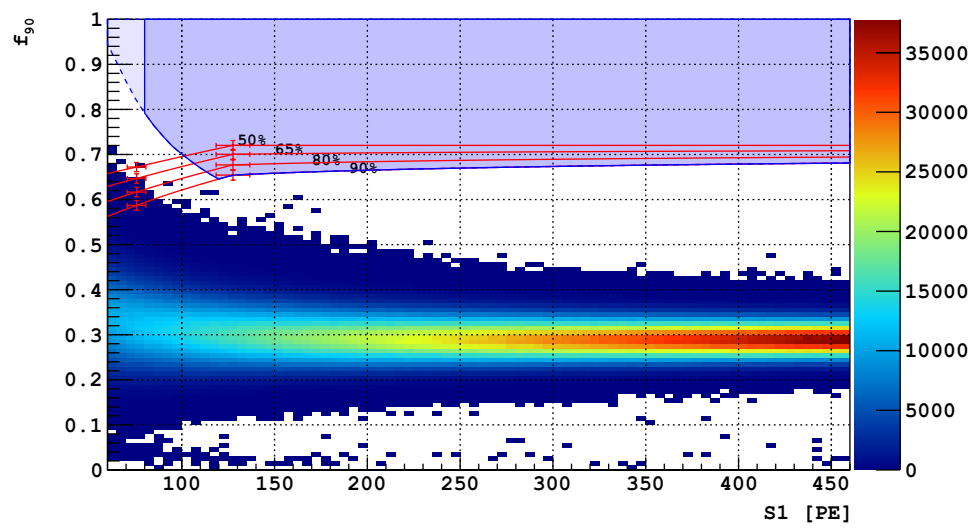

Figure 2. Distribution of the events in the scatter plot of $\mathrm{S} 1$ vs. $f_{90}$ after all quality and physics cuts. Shaded Blue: dark matter search box in the $f_{90}$ vs. S1 plane. Percentages label the $f_{90}$ acceptance contours for nuclear recoils drawn connecting points (shown with error bars) determined from the corresponding SCENE measurements [14]. This picture has been taken from the DS-50 paper [9].

liquid argon on the phototube base divider. This first stage allows us to operate the PMTs at lower gain (typically $4 \times 10^{5}$ ), reducing the occurrence of flashers. The signal undergoes then a second stage that duplicates it into multiple branches. Ultimately, the signal is sent to a set of 12 bit, 250 MSamples/second, digitizers (CAEN 1720). In a similar way, the anodic signals from LSV and WCD phototubes undergo amplification and duplication by means of a custom front-end board. A $\times 10$ amplified signal for each PMT is digitised with NI PXIe5162 National Instruments modules, by sampling them at 1.25 GSamples/second with 10 bit resolution.

An offline reconstruction code is used to analyze the stored waveforms from the TPC and the vetoes photomultiplier tubes. As far as the TPC is concerned, signals from each channel scaled by the corresponding single photoelectron mean are added to form a sum waveform that is used for identifying the pulses in the trigger gate and their start time. Due to the use of DAQ-level zero-suppression, reconstruction of LSV and WCD signals is different from the TPC reconstruction. Pulses are naturally defined as the non-zero portion of each raw waveform for each channel. The DAQ records $20 \mathrm{~ns}$ before and after the waveform rises above and drops below, respectively, the zero-suppression threshold. Each channel is then scaled by the corresponding single photoelectron mean and the channels in each veto detector are summed together. Additional information on the data acquisition, event reconstruction and detector calibrations can be found in $[9,13]$.

\section{Data analysis and WIMP search}

A dark matter search has been performed with data collected with atmospheric argon (AAr) target. This initial AAr data set contains a sample of ${ }^{39} \mathrm{Ar}$ equal to that expected in a campaign of 23 years using UAr at the upper limit of ${ }^{39} \mathrm{Ar}$ activity. The initial dark matter search consists of the data set acquired between November 2013 and May, 2014. We excluded from the data set any runs where one of more of the detectors where not running and where the DAQ, still in a developmental phase, showed signs of instability. We applied a set of data quality cuts, both for the LAr TPC and the vetoes, to exclude backgrounds and misconstructed events. All the details can be found in [9]. We performed a non-blind physics analysis on the 53.4 days 
of WIMP search data. The fiducial mass considered in this analysis $(39.6 \pm 0.6) \mathrm{kg}$. The accurate description of all cuts, their order of application, their effect on livetime, acceptance and fiducial volume, and their systematics and statistical uncertainties can be found in [9]. The total exposure remaining after all cuts prior to the WIMP search box is $(1423 \pm 67) \mathrm{kg}$ d. The distribution of the remaining events in the scatter plot of $f_{90}$ vs. S1 after all quality and physics cuts is shown in Fig. 2. There are $\left(15 \times 10^{6}\right)$ events in this plot, dominated by ${ }^{39} \mathrm{Ar}$ decays. Nuclear recoil acceptance curves in the $f_{90}$ vs. S1 plane were derived from SCENE [14] data and translated to DS-50. $f_{90}$ median values from SCENE, linearly interpolated and assumed to be constant above the highest SCENE NR energy, are translated from the SCENE nuclear recoil energies to DS-50 S1 values. This gives the 50\% contour for DS-50. The other contours and associated errors depend also on the width of the DS-50 $f_{90}$ distributions at each S1, which is established using the same analytical model in use to described the $f_{90}$ spread for ${ }^{39} \mathrm{Ar}$ events described above. These curves are shown in Fig. 2. We observe 4 events passing all TPC cuts and with nuclear-recoil-like $f_{90}$, but with energy depositions in the LSV above our veto cut threshold. In coincidence with one of these 4 neutron candidates, we recorded signals near saturation in both the LSV and the WCD, and therefore we classify that event as a cosmogenic neutron, leaving 3 radiogenic neutron candidates. This number is in agreement with the neutron-induced events passing all TPC cuts expected from radiogenic neutrons from the PMTs based on Monte Carlo studies.

\section{Conclusions}

The DarkSide-50 direct dark matter detection system has completed his first physics data taking at LNGS. An innovative closed-loop argon circulation system with external purification and cooling allows the LAr TPC to achieve an electron drift lifetime of $>5 \mathrm{~ms}$. Photoelectron yield of $(7.9 \pm 0.4) \mathrm{PE} / \mathrm{keV}$ at null field is achieved for detection of the primary argon scintillation, giving the photoelectron statistics necessary for high performance pulse shape discrimination. Figure 2 covers the range of energies from 8.6 to $65.6 \mathrm{keV}$ for ${ }^{39} \mathrm{Ar}$, and a total of $15 \times 10^{6}{ }^{39} \mathrm{Ar} \beta$-decays were recorded over that energy range. Event selection based on the TPC cuts is shown to completely suppress ${ }^{39} \mathrm{Ar}$ background events in the present $(1423 \pm 67) \mathrm{kgd}$ exposure. This exposure contains at least as many ${ }^{39} \mathrm{Ar}$ decays as $215000 \mathrm{kgd}$ of running with underground argon, proving that DS-50 could run for two decades with underground argon and be free of ${ }^{39} \mathrm{Ar}$ background. A WIMP search with the present data-set gives a limit as low as $6.1 \times 10^{-44} \mathrm{~cm}^{2}$, the best result achieved to date with an argon target [9]. The DarkSide program is supported by the NSF and the DOE and by INFN.

\section{References}

[1] P. Benetti et al. (WARP Collaboration), Astopar. Phys. 28, 495 (2008)

[2] M.G. Boulay and A. Hime, Astropart. Phys. 25, 179 (2006)

[3] H.O. Back et al., arxiv:1204.6024

[4] H.O. Back et al., arxiv:1204.6061

[5] H.H. Loosli, Earth Plan. Sci. Lett. 63, 51 (1983)

[6] P. Benetti et al. (WARP Collaboration), Nucl. Inst. Meth. A 574, 83 (2007)

[7] J. Xu et al., arxiv:1204.6011

[8] A. Wright et al., Nucl. Instr. Meth. A 644, 18 (2011)

[9] T. Alexander et al. (DarkSide Collaboration), arXiv:1410.0653

[10] S. Davini, Nucl. Instrum. Meth. A 742 (2014) 183 
[11] T. Alexander et al. (DarkSide Collaboration), JINST 8, C11021 (2013)

[12] C. Aalseth et al., The DarkSide multi-ton detector for the direct dark matter search, Advances in High Energy Physics, Accepted June 2014 (http: / www . hindawi . com/ 4 journals/ahep/raa/541362)

[13] P. Agnes et al. (DarkSide Collaboration), arXiv:1412.2969

[14] T. Alexander et al. (SCENE Collaboration), arxiv:1406.4825 